EPJ Web of Conferences 75, 05008 (2014)

DOI: $10.1051 /$ epjconf/20147505008

(c) Owned by the authors, published by EDP Sciences, 2014

\title{
Size distribution of FeNiB nanoparticles
}

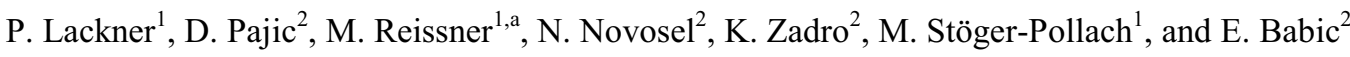 \\ ${ }^{1}$ Institute of Solid State Physics, Vienna University of Technology, A-1040 Vienna, Austria \\ ${ }^{2}$ Department of Physics, Faculty of Science, University Zagreb, HR-10000 Zagreb, Croatia
}

\begin{abstract}
Two samples of amorphous nanoparticles $\mathrm{FeNiB}$, one of them with $\mathrm{SiO}_{2}$ sheath around the core and one without, were investigated by transmission electron microscopy and magnetic measurements. The coating gives mean particle diameters of $4.3 \mathrm{~nm}$ compared to $7.2 \mathrm{~nm}$ for the uncoated particles. Magnetic measurements prove superparamagnetic behaviour above $160 \mathrm{~K}(350 \mathrm{~K})$ for the coated (uncoated) sample. With use of effective anisotropy constant $K_{\text {eff }}$ - determined from hysteresis loops - size distributions are determined both from ZFC curves, as well as from relaxation measurements. Both are in good agreement and are very similar for both samples. Comparison with the size distribution determined from TEM pictures shows that magnetic clusters consist of only few physical particles.
\end{abstract}

\section{Introduction}

Ultrafine magnetic particles are intensively investigated because of their special magnetic properties emerging due to the confined geometry and modified chemical bonding on surfaces. [1,2] Generally, their large surface area and surface energy makes them good candidates for catalysts, as well as for producing media for higher density magnetic recording by special design of their surface through chemical engineering, and for some electromagnetic, environmental and medical applications. [1] However, from fundamental aspects, it is very interesting to investigate the complex magnetism appearing after carefully chosen preparation conditions and composition, leading to different size, saturation of magnetization and magnetic anisotropy. [1,2]

Amorphous magnetic nanoparticles are of special interest in this context, because the absence of crystalline order removes the corresponding anisotropy, and allows to influence stronger the disorder at the surface. [3] Their lower anisotropy than in crystalline state is useful e.g. in enhancement of superconductor properties [4], as well as in other cases where anisotropy is not wanted.
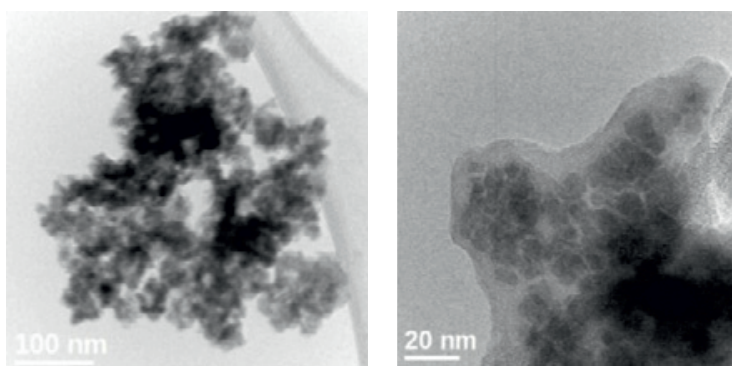

Fig. 1. TEM pictures of $\mathrm{FeNiB}$ (left) and $\mathrm{FeNiB} / \mathrm{SiO}_{2}$ (right).
Transition metal - boron nanoparticles were investigated rarely in the past (much less than spinels and ferrites) in FeNiB samples, concentrate mainly on surface contribution to enhancement of magnetization and anisotropy [5,6], as well as in CoNiB. [3] Here we investigated $\mathrm{FeNiB}$ amorphous particles and the influence of $\mathrm{SiO}_{2}$ coating on their magnetic properties.

\section{Experimental}

Amorphous FeNiB nanoparticles were synthezised by chemical reduction of metallic salts. [7] $\mathrm{Ni}\left(\mathrm{NO}_{3}\right)_{2}$ and $\mathrm{Fe}\left(\mathrm{NO}_{3}\right)_{3}$ were dissolved in ethanol and reducing agent $\mathrm{KBH}_{4}$ was dissolved in water. Immediate addition of $50 \mathrm{ml}$ ethanol containing $0.1 \mathrm{ml}$ tetraetoxysilan to part of the thus obtained material gave $\mathrm{FeNiB}$ particles which are coated with the $\mathrm{SiO}_{2}$ shell. [8]

Structure and composition were investigated by X-ray and FEI TECNAI FZO TEM measurements. A $5 \mathrm{~T}$ MPMS5 SQUID magnetometer from Quantum Design was used for measurement of field and temperature dependence of magnetization in temperature range 2 to $350 \mathrm{~K}$. Time dependence of magnetic moment was measured for up to $30 \mathrm{~min}$ in a QD 9 T PPMS VSM.

\section{Results}

\subsection{Structure}

From X-ray diffraction pure amorphous structure for both compounds is concluded. This is confirmed for the $\mathrm{FeNiB} / \mathrm{SiO}_{2}$ compound by TEM diffraction patterns. In

$\mathrm{a}$

Corresponding author: reissner@tuwien.ac.at

This is an Open Access article distributed under the terms of the Creative Commons Attribution License 2.0, which permits unrestricted use, distribution, and reproduction in any medium, provided the original work is properly cited. 
case of the uncoated FeNiB a fraction of less than $6 \%$ of crystallites with mean diameter of $2.3 \pm 0.2 \mathrm{~nm}$ is present. Figure 1 shows TEM pictures for both samples. Particles agglomerate to uncoated $\mathrm{FeNiB}$ (figure $1 \mathrm{left}$ ). In case of coated FeNiB separation of particles by $\mathrm{SiO}_{2}$ is clearly visible in the HRTEM pictures (figure 1 right). Determination of particle sizes gives log-normal distribution with mean diameter around $4.3 \mathrm{~nm}$ for the coated sample, whereas for the uncoated sample the distribution is more Gaussian-like with a mean diameter of $7.2 \mathrm{~nm}$. EELS measurements indicate that within a few percent deviation the $\mathrm{Fe}$ to $\mathrm{Ni}$ to $\mathrm{B}$ ratio is $1: 1: 1$ in both samples. More than $50 \%$ of the atoms are oxygen. The obtained ratio of the $\mathrm{L} 3$ to $\mathrm{L} 2$ edge peak for $\mathrm{Fe}$ and $\mathrm{Ni}$ was found to be 2.3 and 2.0 for the uncoated FeNiB, whereas for $\mathrm{FeNiB} / \mathrm{SiO}_{2}$ the respective values are $<2$ and $<1.4$. This indicates that both $\mathrm{Fe}$ and $\mathrm{Ni}$ are partly oxidized in $\mathrm{FeNiB}$ but unoxidized in $\mathrm{FeNiB} / \mathrm{SiO}_{2}$. [9]

\subsection{Magnetic measurements}

Due to small size, particles are expected to be magnetically single domains. Magnetic behaviour is therefore expected to show superparamagnetism. [10] Temperature dependence of magnetization was determined both in zero-field-cooled (ZFC) and in fieldcooled (FC) mode for different applied fields (figure 2). Starting temperature was in both cases $2 \mathrm{~K}$, end
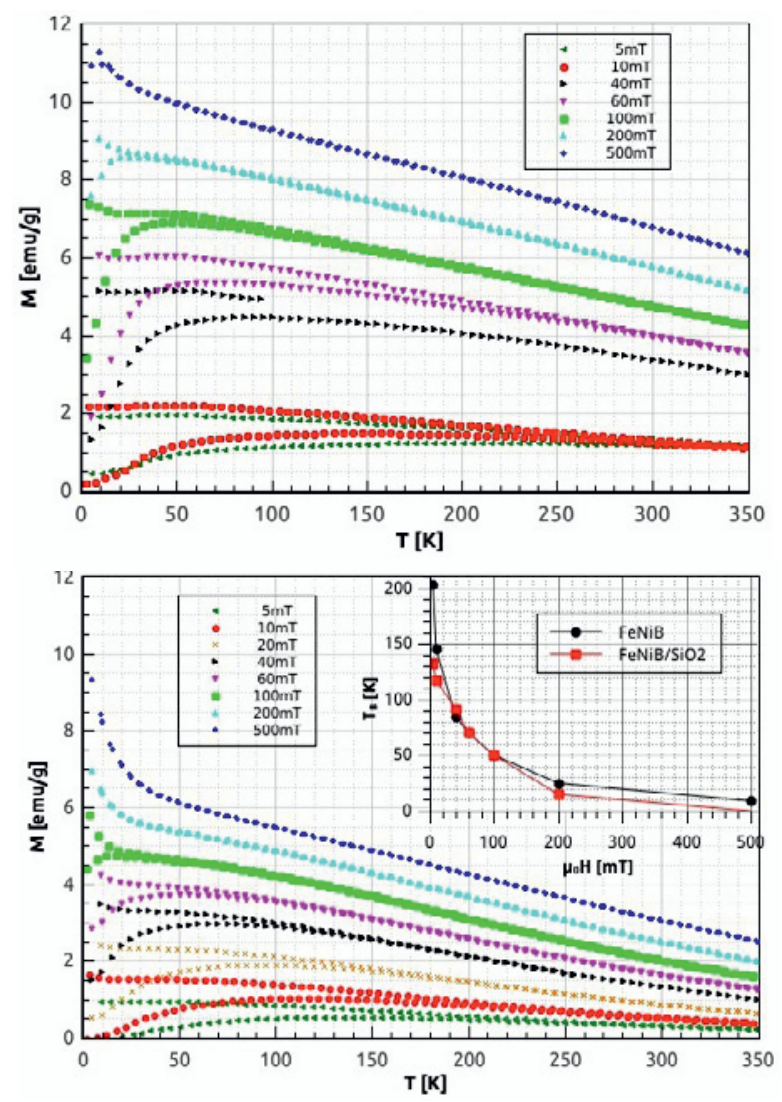

Fig. 2. Temperature dependence of magnetization of $\mathrm{FeNiB}$ (upper picture) and $\mathrm{FeNiB} / \mathrm{SiO}_{2}$ (lower picture) obtained by zero-field-cooled (lower curves) and field-cooled (upper curves) measurements. Inset: field dependence of blocking temperature $T_{\mathrm{B}}$ for both samples. temperature $350 \mathrm{~K}$. Shape of the curve is typical for superparamagnetic behaviour. In low fields both curves show maxima, which shift to lower temperatures with increasing field. Below a distinct temperature $T_{\mathrm{B}}$ splitting of the curves appears. In the superparamagnetic picture $T_{\mathrm{B}}$ can be interpreted as blocking temperature. An extrapolation to zero field gives values of $T_{\mathrm{B}}>350 \mathrm{~K}$ for $\mathrm{FeNiB}$ and $T_{\mathrm{B}} \sim 160 \mathrm{~K}$ for $\mathrm{FeNiB} / \mathrm{SiO}_{2}$.

Hysteresis loops were measured in field range $\pm 5 \mathrm{~T}$ (figure 3). This field values are large enough to reach the reversible part, but no saturation is reached even at highest fields, indicating that a linear term is superposed to a Langevin type trend. From the inner part of the hysteresis (insets figure 3) coercive field $H_{\mathrm{c}}$ is determined. For coated $\mathrm{FeNiB}$ hysteresis becomes fully reversible $\left(H_{\mathrm{c}}=0\right)$ above $160 \mathrm{~K}$, whereas for uncoated compound hysteresis for all temperatures show irreversibility. This is in agreement with the $M(T)$ measurements and shows that hysteresis comes from the irreversibility caused by blocking of superparamagnetic moments below $T_{\mathrm{B}}$. [10]

In full temperature regime, where irreversibilities are present, relaxation measurements were performed by cooling the samples in $10 \mathrm{mT}$ to the target temperature. After field reversal to $-10 \mathrm{mT}$ time dependence of magnetic moment was recorded for $1 \mathrm{~h}$. Resulting curves are in first approximation logarithmic in time (figure 4) allowing the calculation of a logarithmic relaxation rate [1] $S$ from $M(t)=M_{0}-S \ln \left(t-t_{0}\right) . M_{0}$ is the initial
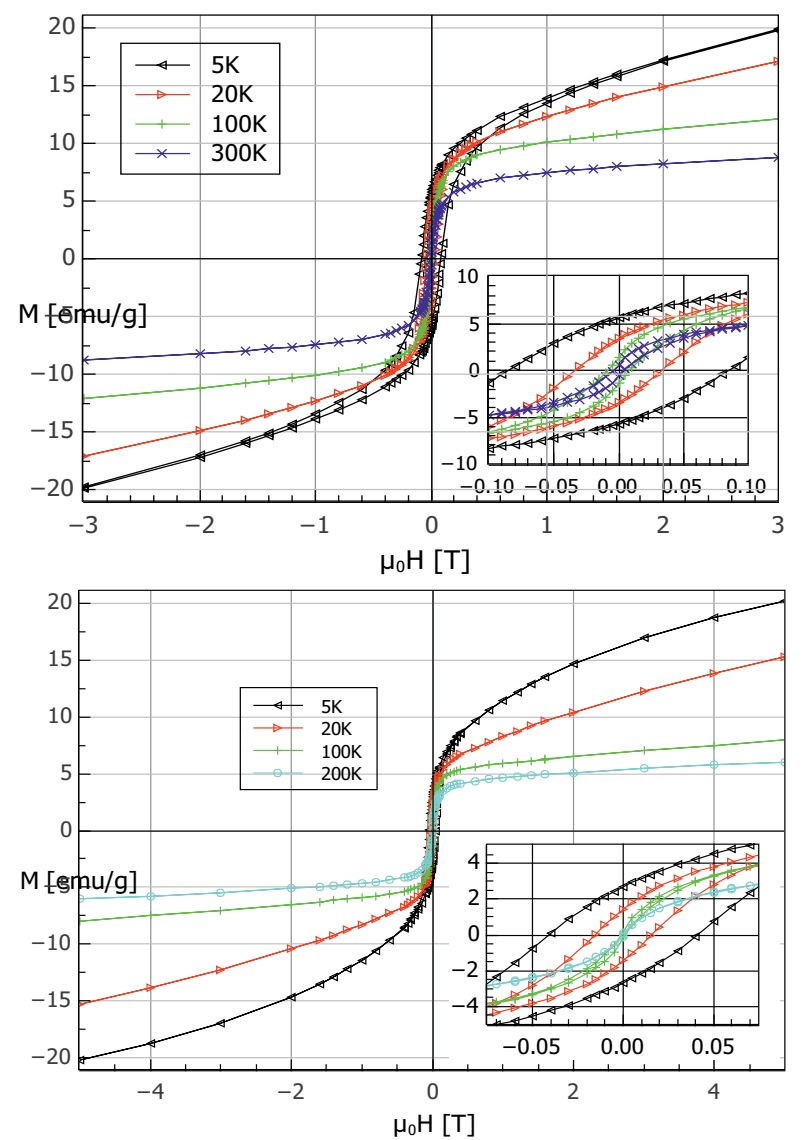

Fig. 3. Field dependence of magnetization of $\mathrm{FeNiB}$ (upper picture) and $\mathrm{FeNiB} / \mathrm{SiO}_{2}$ (lower picture) for selected temperatures. Insets: Enlargement of inner part of hysteresis loops. 


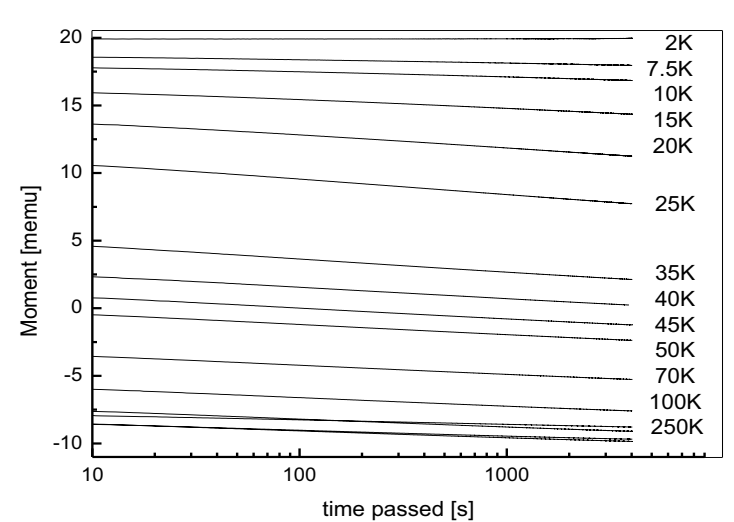

Fig. 4. Relaxation of magnetization for some temperatures for FeNiB sample after cooling in field of $10 \mathrm{mT}$ and reversal at measuring temperature to $-10 \mathrm{mT}$.

magnetization and $t_{0}$ the starting time of relaxation. Temperature dependence of the thus obtained logarithmic relaxation rate [2] is shown in figure 5. A maximum of $S(T)$ appears at $26 \mathrm{~K}$ for both samples. Below this temperature $S$ decreases strongly. Extrapolation to zero temperature gives $S(T)=0$, indicating that relaxation is a pure thermal activated process. [2] The rather flat tail at higher temperatures points to a broad distribution of barrier heights. [2]

\subsection{Magnetic anisotropy and size distribution}

Magnetic anisotropy in nanoparticles is present although their structure is amorphous. It is connected with surface contribution and explains the observed magnetic irreversibility. Application of the Stoner-Wohlfarth model gives the anisotropy density $K=M_{\mathrm{s}} H_{\mathrm{c}} / 2$, where $M_{\mathrm{s}}$ is saturation magnetization and $H_{\mathrm{c}}$ coercive field. [11] Density of $7 \mathrm{gcm}^{-3}$ is used to obtain the volume magnetization for $M_{\mathrm{s}}$. As an approximation, we used maximum magnetization, obtained at temperature of $2 \mathrm{~K}$ in field of $5 \mathrm{~T}$. To obtain the magnetization for the magnetic FeNiB component, we used mass amounts of FeNiB in powder obtained from EELS analysis. The obtained anisotropy density is $K=1.110^{4} \mathrm{Jm}^{-3}$ and $K=9.5 \cdot 10^{3} \mathrm{Jm}^{-3}$, for uncoated and coated particles, respectively. This is considerably lower than for usual crystalline particles of the similar sizes, and comparable to other amorphous nanoparticles. $[2,12,13]$

In case of superparamagnetic nanoparticles it is possible to extract their size distribution from magnetic measurements. Procedure is based on transitions of magnetic moments over the anisotropy barrier, for which the relaxation time is given by the Arrhenius law $\tau=\tau_{0} \exp \left(U / \mathrm{k}_{\mathrm{B}} T\right)$, with $\tau_{0}=10^{-9} \mathrm{~s}$. [2,10] Below blocking temperature $T_{\mathrm{B}}$ the relaxation of particles statistically takes longer than single point measurement (therefore irreversibility is observed), so that the barrier height is obtained as $U=24 \mathrm{k}_{\mathrm{B}} T_{\mathrm{B}} \quad(\tau=30 \mathrm{~s}$ is used for SQUID measurements). Barrier height can be modelled by simple approximation $U=K_{\text {eff }} V$ and from it the characteristic volume of the particles can be calculated when $K_{\text {eff }}$ is obtained from other measurement, e.g. $M(H)$. [11]

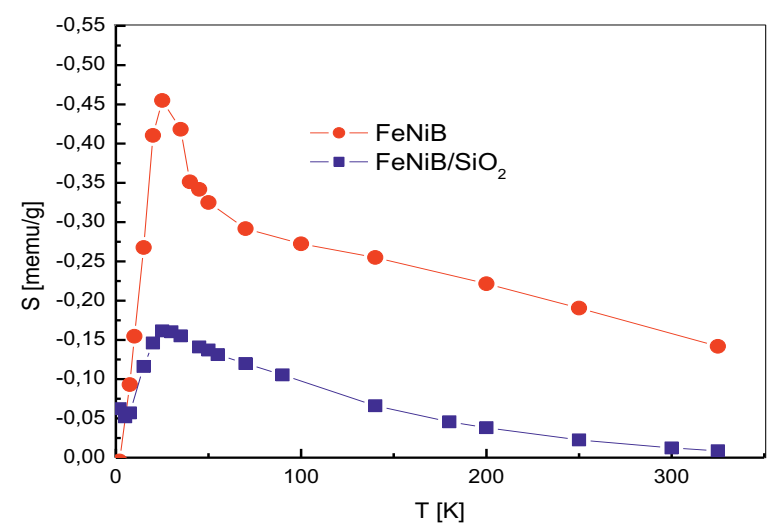

Fig. 5. Temperature dependence of logarithmic relaxation rate $S$ determined from $M(t)=M_{0}-S \ln \left(t-t_{0}\right)$ for coated and uncoated FeNiB samples.

However, in powders always a distribution over barrier sizes is present. Since ZFC $M(T)$ measurements count how many magnetic moments are unblocked up to temperature $T$, i.e. for what amount of magnetization this $T$ is equal to $T_{\mathrm{B}}$, the distribution of blocking temperatures in the sample is revealed as $f\left(T_{\mathrm{B}}\right) \sim \mathrm{d}\left(T \cdot M_{\mathrm{ZFC}}(T)\right) / \mathrm{d} T$ (for details see [2,14]). Assuming spherical particles, $T_{\mathrm{B}} \sim D^{3}$, so that $f(T) \sim \mathrm{d}\left(T \cdot M_{\mathrm{ZFC}}(T)\right) / \mathrm{d} D \cdot \mathrm{d} D / \mathrm{d} T$ gives the distribution over diameter $f(D)=\mathrm{d}\left(T \cdot M_{\mathrm{ZFC}}(T)\right) / \mathrm{d} T \cdot T^{2 / 3}$, where diameter is given by $D=\left(6 \cdot 24 \mathrm{k}_{\mathrm{B}} \mathrm{T} / \pi K\right)^{1 / 3}$.

Taking the obtained effective anisotropy density $K_{\text {eff }}$ from $M(H)$ analysis, the calculated $f(D)$ distribution is shown in figure 6.

The distribution of magnetic particles over volumes can also be obtained from the performed relaxation measurements. Parameter $S$ tells how many particles relax, while measuring at a specific temperature. [15] Therefore, after transforming the temperature domain of $S(T)$ to diameter domain in $S(D)$ using the same procedure as above, the distribution of particles over diameter is obtained and also shown in figure 6.

This procedure was successful in determination of magnetic unit sizes in different systems, from magnetic nanoparticles $[14,15,16]$, to magnetic clusters in amorphous ribbon [17] and magnetic nano-regions in multiferroics. [18] Therefore, we believe this model is valid in case of presented amorphous nanoparticles.

\section{Discussion}

In both compounds, consistency is found between $M(T)$ and $M(H)$ curves, regarding their observed irreversibility ranges. Normalizing the magnetization to mass of FeNiB present in powders, the maximum magnetization at same conditions $(2 \mathrm{~K}, 5 \mathrm{~T})$ is $30.6 \mathrm{emu} / \mathrm{g}$ for uncoated, and $44 \mathrm{emu} / \mathrm{g}$ for coated $\mathrm{FeNiB}$ particles. Therefore coating with $\mathrm{SiO}_{2}$ leads to higher magnetic order. The temperature where irreversible behaviour appears is higher for the uncoated than for the coated sample, showing that anisotropy barriers are higher in uncoated case than in coated within the framework of blocking of superparamagnetic particles.

Geometric determination of the particle size distribution from TEM pictures give slightly different 

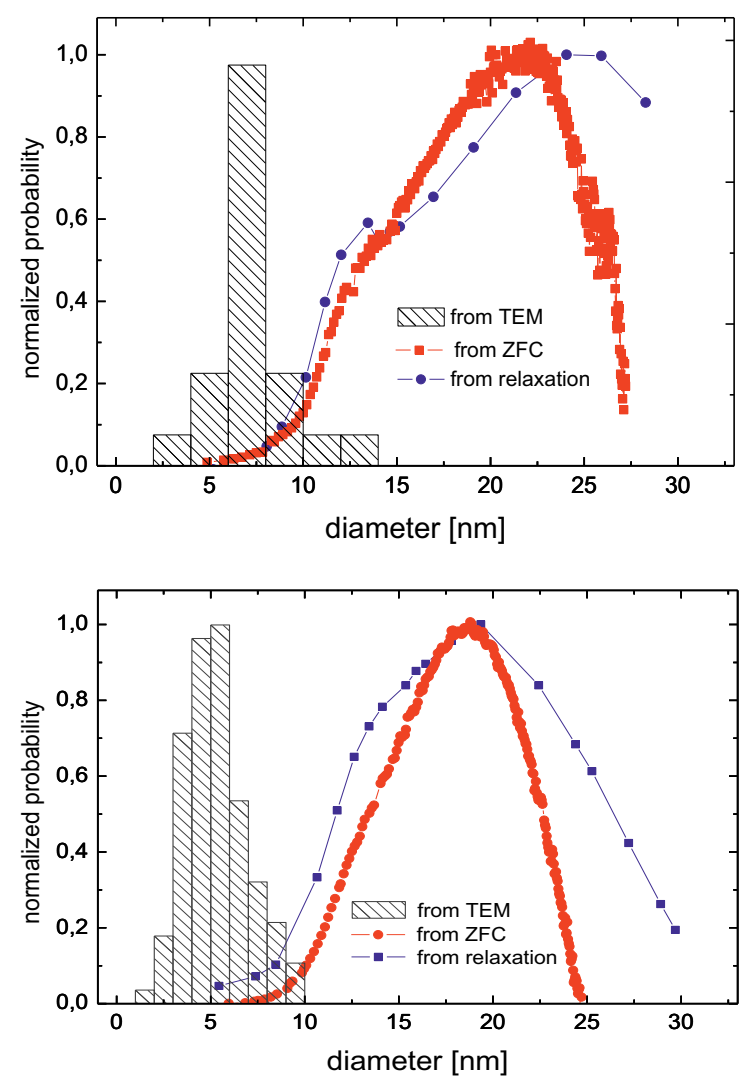

Fig. 6. Particle size distributions obtained geometrically from TEM pictures, and magnetically from ZFC-curves, as well as from magnetic relaxation data for uncoated $\mathrm{FeNiB}$ (upper panel) and coated $\mathrm{FeNiB} / \mathrm{SiO}_{2}$ (lower panel).

results for the two samples. For the uncoated FeNiB a Gaussian-like distribution with a mean value of $7.2 \mathrm{~nm}$ is obtained, whereas for the coated $\mathrm{FeNiB} / \mathrm{SiO}_{2}$ compound a log-normal distribution with smaller mean particle size of $4.3 \mathrm{~nm}$ is found (figure 6). If those diameters are used, the effective magnetic anisotropy density calculated as $K_{\text {eff }}=U / V$ where $U=24 k_{\mathrm{B}} T_{\mathrm{B}}$, comes in the range of $10^{6} \mathrm{Jm}^{-3}$, which is unrealistically high, even impossible for amorphous materials. [2,12,13] Therefore, $K_{\text {eff }}$ obtained from $M(H)$ is used as realistic one, and analysis performed as described above. Magnetically determined particle size distributions are for both samples at higher mean values than the geometrically determined ones, indicating that several particles are magnetically coupled, forming magnetic clusters. Magnetically determined mean grain size distributions do only slightly differ in mean value and shape. For both, the centre of gravity of the distribution function is around $19 \mathrm{~nm}$. This implies that although the grains are better separated due to the $\mathrm{SiO}_{2}$ sheath, magnetic coupling between particles still appears. This is caused by the lower anisotropy energy of the coated sample, which allows easier reorientation in applied field and the larger magnetization of this sample. The latter fact also indicates, that in the coated particles surface sheath is either thinner than for the uncoated particles and/or there is less moment disorder at the surface.

To summarize, we have investigated two samples of amorphous $\mathrm{FeNiB}$ nanoparticles, where in one sample the particles are coated by $\mathrm{SiO}_{2}$ sheath. This coating gives physically smaller particles, enlarged superparamagnetic region, and higher magnetic moments. Determination of anisotropy energy constant $K_{\text {eff }}$ from the hysteresis loops gives a slightly higher value for the uncoated sample. Using these $K_{\text {eff }}$ values magnetic grain size distributions were determined, which are for both samples very similar. Comparison with the geometrically determined distribution indicate that the magnetic units consist of only small number of physical nanoparticles.

\section{Acknowledgement}

We thank ÖAD and Croatian Ministry of Science for supporting this work through research project within Austrian-Croatian bilateral scientific cooperation.

\section{References}

1. S. P. Gubin, Yu A. Koksharov, G. B. Khomutov, G. Yu Yurkov, Russian Chemical Reviews 74, 489 (2005)

2. E.M. Chudnovsky, J. Tejada, Macroscopic Quantum Tunneling of the Magnetic Moment (Cambridge University Press, Cambridge, UK, 1998)

3. B. Molina Concha, R. D. Zysler, H. Troiani, H. Romero, Physica B 354, 121 (2004)

4. N. Novosel, S. Galić, D. Pajić, Ž. Skoko, I. Lončarek, M. Mustapić, K. Zadro, E. Babić, Supercond. Sci. Technol. 26, 065004 (2013)

5. E. De Biasi, C. A. Ramos, R. D. Zysler, H. Romero, Phys. Rev. B 65, 144416 (2002)

6. E. De Biasi, R. D. Zysler, C. A. Ramos, H. Romero, Physica B 320, 203 (2002)

7. S. Wells, S. W. Charles, S. Mørup, S. Linderoth, J. van Wonterghem, J. Larsen, M.B. Madsen, J. Phys.: Condens. Matter 1, 8199 (1989)

8. C. Saiyasombat, N. Petchsang, I. M. Tang, J. H. Hodak, Nanotechnology 19, 085705 (2008)

9. H. Tan, J. Verbeeck, A. Abakumov, G. Van Tendeloo, Ultramicroscopy 116, 24 (2012)

10. C. P. Bean, J. D. Livingston, J. Appl. Phys. 30, 120S (1959)

11. E. C. Stoner and E. P. Wohlfarth, Phil. Trans. R. Soc. Lond. A 240, 599 (1948)

12. R. D. Zysler, H. Romero, C. A. Ramos, E. De Biasi, D. Fiorani, J. Magn. Magn. Mater. 266, 233 (2003)

13. M. Mustapić, D. Pajić, N. Novosel, E. Babić, K. Zadro, M. Cindrić, J. Horvat, Ž. Skoko, M. Bijelić, A. Shcherbakov, Croat. Chem. Acta 83, 275 (2010)

14. R. S. DiPietro, H. G. Johnson, S. P. Bennett, T. J. Nummy, L. H. Lewis, D. Heiman, Appl. Phys. Lett. 96, 222506 (2010)

15. O. Iglesias, F. Badia, A. Labarta , L. I. Balcells, Z. Phys. B 100, 173 (1996)

16. D. Pajić, K. Zadro, R. E. Vandenberghe, I. Nedkov, J. Magn. Magn. Mater. 281, 353 (2004)

17. D. Pajić, K. Zadro, R. Ristić, I. Živković, Ž. Skoko, E. Babić, J. Phys.: Condens. Matter 19, 296207 (2007)

18. D. Pajić, Z. Jagličić, Z. Trontelj, J. Appl. Phys. 112, 073908 (2012) 